\title{
PRE within Ss: Conventional effect on differential speeds, reverse effect on choices'
}

NORMAN E. SPEAR AND JOSEPH H, SPITZNER

RUTGERS UNIVERSITY

Rats were given equal experience with alternatives which were either continuously or partially reinforced. A reduction in the stimulus overlap between discriminanda increased the tendency toward a conventional PRE with in $S s$ in terms of differential speeds; but on free-choice trials, all Ss more frequently chose the previously continuous-reinforcement alternative, and this was uniform across conditions.

When the effect of percentage reinforcement on extinction has been measured within Ss-by comparing relative running speeds in extinction to a stimulus previously associated with partial reinforcement (PRF) and another previously associated with continuous reinforcement (CRF)-the results have been quite different from those obtained with the more common between-S design. The former design has shown that $S$ responds to a stimulus previously associated with CRF with no less, or even greater, resistance to extinction than to a stimulus previously associated with PRF (for example, Spear, 1964; Amsel, 1964; Pavlik, Carlton, \& Hughes, 1965). In addition, a uniform increase in resistance to extinction of responding to both CRF and PRF has accompanied this within-S PRE. While this may indicate that the effect of a given percentage reinforcement is generalized across discriminanda (due to common stimulus elements), Spear \& Spitzner (1967) have suggested mediated generalization (cf., Amsel, 1964) as a more likely cause.

The basic purpose of the present experiment was to test directly the role of primary stimulus generalization in determining these phenomena. Specifically, the present experiment compared running speeds by $S$ to PRF (33 percent) and CRF (100 percent) alternatives of a $T$ maze under one of three discrimination tasks-a brightness discrimination with position irrelevant (Group 100-33B), a position discrimination with brightness irrelevant (Group 100-33P), or a discrimination task in which brightness and position both were perfectly correlated with percentage reinforcement (Group 100-33BP). It was predicted that Ss in Group 100-33BP would have a greater tendency toward a conventional within-S PRE, and lesser tendency toward a uniform increase in resistance to extinction across alternatives, compared to $\mathrm{Ss}$ in $100-33 \mathrm{~B}$ or $100-33 \mathrm{P}$, since the latter groups would have greater stimulus overlap between alternatives. Method

Subjects and apparatus. The Ss were 40 naive female albino rats, 180-210 gm, 55-65 days old, purchased from Sprague-Dawley Co. of Madison, Wis. The $T$ maze had a movable stem permitting convenient inter- change of the position of a given brightness (black vs. white). The doors just beyond the choice point were used for forcing purposes and to prevent retracing.

Design and procedure. Five groups of eight Ss each included Groups 100-100 and 33-33 (100 percent or 33 percent reinforcement, respectively, occurred in both alternatives of the $\mathrm{T}$ maze; position of a given brightness was varied randomly from trial to trial as in conditions 100-33B and 100-33P) and Groups 100-33BP, 100-33B, and 100-33P described above. Each of the latter three groups had one alternative associated with CRF and the other with PRF。

Following prehandling and adjustment to the 23-hr. schedule of food deprivation (described by Spear, 1964) for nine days, Ss were run six trials per day for six days each week-acquisition for 55 days ( 330 trials) and extinction for 15 days (90 trials). The Ss were run in a rotation containing one member from each group resulting in an intertrial interval of about $12 \mathrm{~min}$. On each reinforced trial, $\mathrm{S}$ consumed six 45-mg Noyes pellets.

Each day the first trial was free, the second was forced to the alternative opposite that chosen (in terms of the relevant dimension) and the remaining four trials were forced half to each alternative according to a predetermined, random schedule. Extinction differed from acquisition only in terms of the absence of reward pellets. Running speeds (ft. per sec.) relevant to this paper are the committed-start (covering a distance of 6 in. beginning 6 in. beyond the choice point) and committed-goal speeds (last $2-1 / 2 \mathrm{ft}$. in each arm to a point 2 in. before the masked reward container) taken from the randomly forced trials. Results

Acquisition. During the last five days of acquisition, only one "error" was made among Ss choosing between differential percentage reinforcement and that by an $S$ in Group 100-33B. In addition, no reliable $(p<.05)$ differences in terms of committed-start speed were obtained during the last five days of acquisition either within Ss or between Groups 100-33BP, 100-33B, and 100-33P. As found in the straight runway, the effect of percentage reinforcement was greater nearer the goal in both acquisition and extinction. Thus committedgoal speed was greater within Ss to CRF than PRF, $F=26.23, d f=1 / 21, p<.001$, and this difference was uniform across Groups 100-33BP, 100-33B, and 100-33P (the interaction between alternatives and groups yielded an $F$ less than one). During the last five days of acquisition, committed-start speed was reliably greater for $\mathrm{Ss}$ in Group 100-100 than in 33-33, F=4.68, $\mathrm{df}=$ 


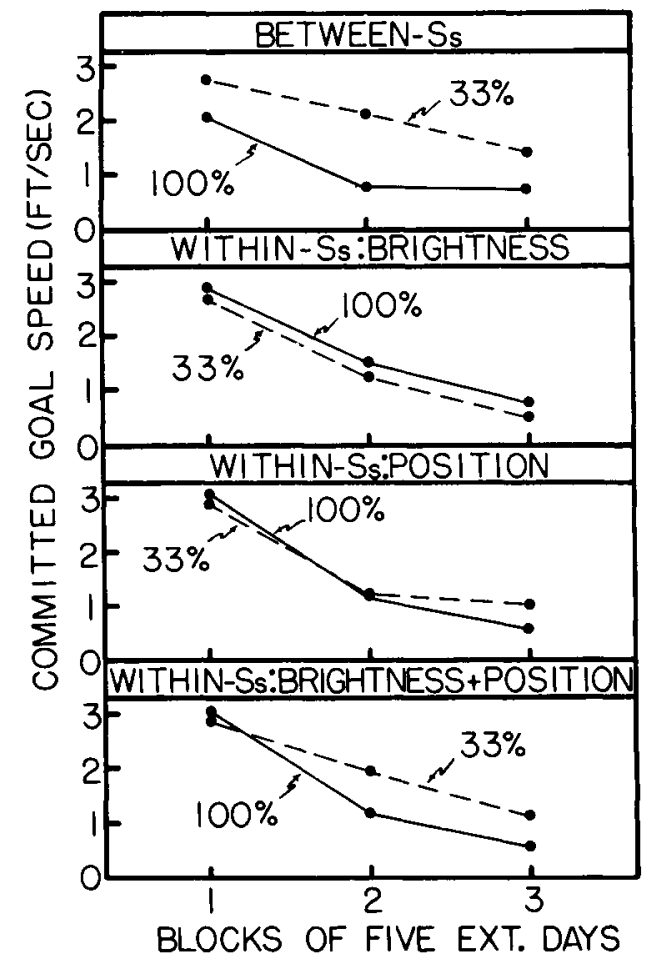

Fig. 1. Mean committed-goal speed during each block of five extinction days. The between-Ss PRE (top panel of figure) is defined in terms of data from Groups 100-100 and 33-33.

$1 / 14, \mathrm{p}<.05$; but as within $\mathrm{Ss}_{\text {, }}$ this effect was stronger in terms of committed-goal speed, $F=17.48, \mathrm{df}=1 / 14$, $\mathrm{p}<.001$

Extinction. It may be seen in Fig. 1 that the usual between-S PRE occurred-mean committed-goal speed in Group 33-33 was greater than that in Group 100-100 throughout Extinction Days 1-15, 6-10, or 11-15 as reflected by the significant $F$ values $(d f=1 / 14)$ of $46.04,48.30$, and 9.94 , respectively. The more important result for this experiment is the consistent interaction between type of discrimination and alternative (see Fig. 1). This interaction reflected the dependence of the differential in running speed upon type of discrimination and was reliable whether analyzed on Extinction Days 1-15, 6-10, or 11-15, $\mathrm{F}=5.50$, 3.92 , and 4.16 , respectively $(\mathrm{df}=1 / 21, \mathrm{p}<.05)$. The Ss in Group 100-33B showed the typical result of continued greater running speed to their CRF alternative throughout extinction, although this difference did not attain statistical reliability. In contrast, Ss in Group BP had greater committed-goal speed to the PRF than the CRF alternative-a conventional PRE within Ss-whether estimated in terms of Extinction Days 1-15,6-10, or 11-15 $(t=2.80,2.86$, and 2.57, df $=21, p<.05$, respectively $)$. Similarly, although mean committed-goal speed by Group $\mathrm{P}$ was about equal to the CRF and PRF alternatives during Extinction Days 6-10, this group also showed a conventional PRE within Ss during Extinction Days $11-15(t=2.16, d f=21, p<.05)$.

In contrast to the speed data, the mean number of choices of the formerly CRF alternative did not differ among Groups 100-33BP, 100-33B and 100-33P; whether in terms of Extinction Days 1-15, 6-10, or 11-15, $F$ values were less than one. Moreover, each of these three groups had only one $\mathrm{S}$ which chose the previously PRF alternative with greater frequency than the CRF alternative during the last ten days of extinction.

\section{Discussion}

This experiment has demonstrated that resistance to extinction of responding after PRF is greater than that after CRF both within Ss and between Ss; but the effect within Ss depends upon the extent of stimulus overlap between the discriminanda. When only brightness was perfectly correlated with percentage reinforcement but position occurred equally often in association with CRF and PRF (Group 100-33B), a PRE did not occur within Ss; but a conventional PRE did occur within Ss when both brightness and position were perfectly correlated with percentage reinforcement (Group 100-33BP). Moreover, this was true even though both differential speed and choice behavior in late acquisition indicated that the site of a given percentage reinforcement was discriminated as well in the former group as in the latter. The fact that Group 100-33P was apparently an intermediate case may be explained in that the position dimension contains extramaze cues as well as proprioceptive cues providing greater stimulus control than the brightness dimension.

It is notable that choice behavior did not differ among Groups 100-33BP, 100-33B and 100-33P during extinction, in spite of the considerable deviation among these groups in terms of differential speed within Ss. In fact, $S$ s in Groups 100-33BP and 100-33B continued to run to the formerly CRF alternative on free choice trials even though they had greater forced-trial running speeds to their previously PRF alternative. Related data suggest that this inverse relationship was not an artifact of receiving the free choice only on the first trial of each day-(1) There were no systematic changes in the running-speed relationships from the beginning to the end of the daily session of trials, and (2) When a free trial has been given both early and late in a daily session (for example, Spear, 1964; Spear \& Spitzner, 1967) equivalent results have been obtained with either trial.

\section{References}

Amsel, A. Partial reinforcement acquisition and extinction effects under within-subject and between-subject conditions. Paper presented at Psychonomic Society Meeting, Niagara Falls, 1954.

Pavlik, W. B., Carlton, P. L., \& Hughes, R. A. Partial reinforcement effects in a runway: Between and within-subjects. Psychon. Sci., 1965, 3, 533-534.

Spear, N. E. Choice between magnitude and percentage of reinforcement. J. exp. Psychol., 1964, 68, 44-52.

Spear, N. E., \& Spitzner, J. H. PRE in a T-maze brightness discrimination within and between Ss. J. exp. Psychol., 1967, in press.

\section{Note}

1. This research was supported by grants (MH-08888 and MH-12064) from the National Institute of Mental Health. 